\title{
Ultrastructural alterations in Schistosoma mansoni juvenile and adult male worms after in vitro incubation with primaquine
}

\author{
Reem Osama A Kamel, Fatma El-Zahraa Anwar Bayaumy/ ${ }^{+}$ \\ Ain Shams University, Women College for Arts, Science and Education, Department of Zoology, Asmaa Fahmey St., Cairo, Egypt
}

BACKGROUND Praziquantel has been cited as the only drug for treating schistosomiasis. However, concerns over drug resistance have encouraged the search for novel drug leads. The antimalarial drug primaquine possesses interesting anti-schistosmal properties.

OBJECTIVES This study is the first to document the potential role of primaquine as a schistosomicide and the ultrastructural changes induced by primaquine on juvenile or adult male worms of Schistosoma mansoni.

METHODS Ultrastructural alterations in the tegumental surface of 21-day-old juvenile and adult male worms of $S$. mansoni were demonstrated following primaquine treatment at different concentrations $(2,5,10,15$, and $20 \mu \mathrm{g} / \mathrm{mL})$ and incubation periods $(1$, $3,6,24$, and $48 \mathrm{~h}$ ) in vitro, using both scanning and transmission electron microscopy.

FINDINGS At low concentrations $(2,5$, and $10 \mu \mathrm{g} / \mathrm{mL})$ both juvenile and adult male worms were alive after $24 \mathrm{~h}$ of incubation, whereas contraction, paralysis, and death of all worms were observed after $24 \mathrm{~h}$ of drug exposure at $20 \mu \mathrm{g} / \mathrm{mL}$. The tegument of juvenile and adult male worms treated with primaquine exhibited erosion, peeling, and sloughing. Furthermore, extensive damage of both tegumental and subtegumental layers included embedded spines, and shrinkage of muscles with vacuoles. The in vitro results confirmed that primaquine has dose-dependent effects with $20 \mu \mathrm{g} / \mathrm{mL}$ as the most effective concentration in a short incubation period.

MAIN CONCLUSIONS The schistosomicidal activity of primaquine indicates that this drug possesses moderate in vitro activity against juvenile and adult male worms, since it caused high mortality and tegumental alterations. This study confirmed that the antimalarial drug primaquine possesses anti-schistosomal activity. Further investigation is needed to elucidate its mechanism of action.

Key words: Schistosoma mansoni - primaquine - Praziquantel - schistosomicidal activity - tegumental alteration

Schistosomiasis is a chronic disease with high prevalence and wide distribution worldwide. It is endemic in 76 countries distributed throughout Africa, Southeast Asia, and Central and South America (Mata-Santos et al. 2014). It affects more than 200 million people worldwide, and nearly 800 million others are at risk of infection (Colley et al. 2014).

Due to unavailability of a schistosomiasis vaccine, the common strategy for treatment and control is mainly based on chemotherapy (Pereira et al. 2015). Praziquantel (PZQ) is the drug of choice for large-scale treatment of schistosomiasis as it is a safe and cheap therapy (Cheng et al. 2011). The drug targets the adult worm, but has minor activity against the young developing stages (i.e., schistosomula); hence, repeated treatment is necessary to kill the parasites that have matured (Doenhoff \& Pica-Mattoccia 2006). Unfortunately, drug resistance is still a threat in long-term administration of PZQ. Therefore, new chemotherapies against schistosomiasis are desperately required (Doenhoff et al. 2008).

Both anti-malarials and their derivatives may have a therapeutic impact in early and chronic schistosomiasis (Mitsui \& Aoki 2010). Interestingly, the antimalarial

\footnotetext{
doi: 10.1590/0074-02760160324

+ Corresponding author: fatmaanwar1980@gmail.com

Received 16 July 2016

Accepted 15 December 2016
}

drug, mefloquine, is reported to be effective against both Schistosoma mansoni and Schistosoma japonicum in vivo and in vitro (Manneck et al. 2010, Mossallam et al. 2015). Furthermore, it was demonstrated that not only mefloquine, but also other antimalarial drugs such as amodiaquine, primaquine and chloroquine exhibited in vitro anti-schistosomal activity. In addition, schistosomiasis and malaria share roughly the same epidemic regions (Mitsui \& Aoki 2010). Thus, it has been confirmed that the anti-malarial drug primaquine displays antischistosomal properties against both juvenile and adult worms of $S$. mansoni in vitro, causing a pronounced deformation of the parasite body (Holtfreter et al. 2011). Furthermore, primaquine was found to affect the lysosomal acidic vesicles of the schistosomula involved in endocytosis and detoxification (Carneiro-Santos et al. 2001). Moreover, Mitsui and Aoki (2010) reported that primaquine inhibited the daily egg output and decreased the survival of both male and female worms.

The tegument of an adult schistosome is a protective sheath that plays a role in defence as well as in the uptake of nutrients, osmoregulation, and excretion. Although alterations in the surface ultrastructure of schistosome worms have received little attention, several investigators have used them for evaluating anti-schistosomal drugs (El-Shabasy et al. 2015). As male worms are more sensitive to anti-schistosomal drugs than females are (Shaw \& Erasmus 1987), the current study is the first to document the alterations in the tegumental surface of 
21-day-old juvenile and adult male worms of $S$. mansoni following primaquine treatment at different concentrations in vitro by a detailed temporal examination using both scanning and transmission electron microscopy.

\section{MATERIALS AND METHODS}

Animals and parasites - Male Syrian golden hamsters (Mesocricetus auratus) weighing 100-110 g were infected via shaved abdominal skin with $350 \pm 10$ cercariae of the Egyptian strain of S. mansoni per animal, freshly shed from experimentally infected Biomphalaria alexandrina. The animals and parasites were obtained from the Schistosome Biological Supply Centre (SBSC), Theodor Bilharz Research Institute (TBRI), Giza, Egypt. The hamsters were maintained on a standard commercial pellet diet and kept in an air-conditioned animal house at $20-22^{\circ} \mathrm{C}$. All experiments were approved by the animal ethics committee and carried out at the SBSC/TBRI, in accordance with the internationally valid animal ethics guidelines.

Drugs - Primaquine bisphosphate and Praziquantel were purchased from Sigma-Aldrich Chemical Co. (St. Louis, MO, USA). Thirty milligrams of primaquine was dissolved in $3 \mathrm{~mL}$ injectable water to prepare a stock solution of $10 \mathrm{mg} / \mathrm{mL}$ (Holtfreter et al. 2011). Further dilutions were carried out using aqua ad injectabilia. PZQ was dissolved in $0.1 \%$ dimethyl sulphoxide (DMSO) to be used as a reference drug.

Culture medium and parasite preparation - Juvenile worms were recovered from the hepatic veins of infected hamsters after 21 days post infection by perfusion (Clegg $\&$ Smithers 1972). Seven weeks following infection, adult $S$. mansoni worms were retrieved by perfusion of both the hepatic portal system and the mesenteric veins (Duvall \& DsWitt 1967). All the recovered juvenile and adult male worms were washed several times from blood in small sieves (20 $\mu \mathrm{M}$ mesh) using phosphate buffer. They were then rinsed thrice with the culture medium used for the assay in a sterilised laminar flow chamber. The culture medium used was Roswell Park Memorial Institute medium (RPMI 1640) supplemented with $2 \mathrm{mM} \mathrm{L-glutamine,}$ $20 \%$ foetal calf serum, and antibiotics ( $300 \mu \mathrm{g}$ streptomycin, $300 \mathrm{IU}$ penicillin, and $160 \mu \mathrm{g}$ gentamycin per $\mathrm{mL}$ ).

Assessment of the anti-schistosomal effect of primaquine on $S$. mansoni juvenile or adult male worms in vitro - Juvenile or adult male worms of $S$. mansoni were subjected to treatment with varying concentrations of primaquine $(2,5,10,15$, and $20 \mu \mathrm{g} / \mathrm{mL})$. These concentrations were based on the plasma concentration levels during chemotherapy and prophylaxis (Elmes et al. 2006). The bioassay was carried out in 24 -well culture plates (Costar) containing the same medium as previously mentioned (Xiao et al. 2014). Four juvenile or adult male worms were used in each well and two replicates were set up. Subsequently, the plates were incubated in a $5 \% \mathrm{CO}_{2}$ incubator at $37^{\circ} \mathrm{C}$ for $24 \mathrm{~h}$ (Xiao et al. 2007). The final volume in each well was $2 \mathrm{~mL}$. Pure medium served as the negative control, whereas PZQ $(5 \mu \mathrm{g} / \mathrm{mL})$ was used as the positive control. Treated worms were examined for viability at intervals of $1,3,6,24$, and $48 \mathrm{~h}$ using an Olympus Inverted Microscope (Tokyo, Japan). The surviving parasites were identified as freely swimming without any structural deformation, whereas the dead parasites were recognised by complete loss of motility and were lying at the bottom of the well (Moraes 2012). The mortality rate of the worms was recorded for each concentration by calculating the number of dead worms relative to the total number of worms (Holtfreter et al. 2011, El Bardicy et al. 2012). The mean \pm SD of three mortality values (of three independent experiments) for each concentration was also calculated at different times.

Scanning electron microscopy of juvenile and adult male worms of $S$. mansoni incubated for 24 h with PZQ or primaquine - Scanning electron microscopy (SEM) was used for the ultrastructural analysis of the effect of primaquine bisphosphate against $S$. mansoni juvenile or adult male worms. The juvenile or adult male worms incubated in pure medium (negative control), $5 \mu \mathrm{g} / \mathrm{mL}$ of PZQ (positive control), or those incubated with different concentrations $(10,15$, and $20 \mu \mathrm{g} / \mathrm{mL})$ of primaquine for $24 \mathrm{~h}$, were washed several times with normal saline, fixed with $2.5 \%$ glutaraldehyde, and then dehydrated with serial dilutions of ethanol using an automatic tissue processor (Leica EM TP). Thereafter, the worms were dried using a $\mathrm{CO}_{2}$ critical point drier (Tousimis Audosamdri- 815). Specimens were coated with a gold sputter coater (SPI- Module). Coated worms were observed and photographed using a scanning electron microscope (JEOL- JSM - 5500 LV) under the high vacuum mode (Xavier et al. 2010) at the Regional Center of Mycology and Biotechnology, El Azhar University, Cairo, Egypt.

Transmission electron microscopy of adult male worms of S. mansoni incubated for $24 \mathrm{~h}$ with PZQ or primaquine - Primaquine bisphosphate-treated $S$. mansoni adult male worms at different concentrations $(10,15$ and $20 \mu \mathrm{g} / \mathrm{mL})$ and other adult male worms incubated in pure medium (negative control) or in $5 \mu \mathrm{g} / \mathrm{mL}$ PZQ (positive control) for $24 \mathrm{~h}$, were fixed in $5 \%$ glutaraldehyde prepared in $0.1 \mathrm{M}$ sodium cacodylate buffer for $4 \mathrm{~h}$. Post fixation was performed with $1 \%$ osmium tetroxide in the same buffer for $2 \mathrm{~h}$. Afterwards, the worms were dehydrated through a graded ethanol series before embedding in an epoxy resin. Ultrathin sections (50-80 nm thick) were double stained with uranyl acetate followed by lead citrate. The stained sections were examined and photographed with a JEOL 1010 Transmission Electron Microscope (Xavier et al. 2014) at the Regional Centre for Mycology and Biotechnology, Al-Azhar University, Cairo, Egypt.

Statistical analysis - The results of this study were calculated as mean \pm SD using Microsoft Excel software.

\section{RESULTS}

Effect of primaquine on the mortality rate of S. mansoni juvenile and adult male worms in vitro - The mortality rate of both juvenile (21 days old) and adult male worms (seven weeks old) at different incubation periods $(1,3,6,24$, and $48 \mathrm{~h})$ with different concentrations $(2,5$, 10,15 , and $20 \mu \mathrm{g} / \mathrm{mL}$ ) of primaquine were studied. As expected, all the worms (juvenile and adult male) incu- 
bated in pure medium (negative control) exhibited active movement and were alive throughout the experimental period of $48 \mathrm{~h}$. The positive control group exposed to

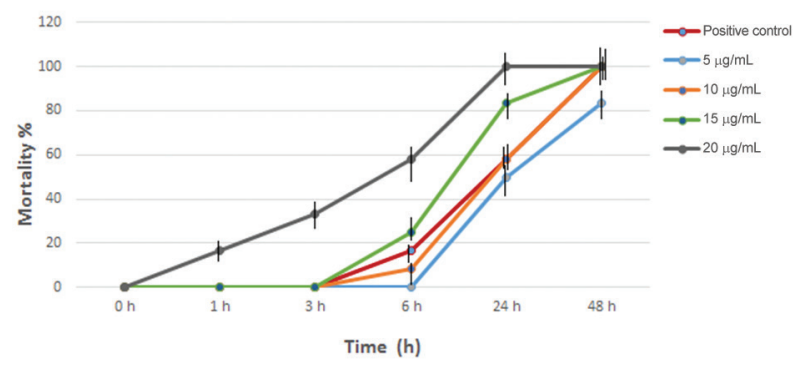

Fig. 1: mortality rate (\%) of Schistosoma mansoni juvenile worms treated in vitro with different concentrations of primaquine for different incubation times. The mortality rate of both the negative control group and the group treated with $2 \mu \mathrm{g} / \mathrm{mL}$ of primaquine was $0 \%$. Error bars represent the \pm SD from three independent experiments.

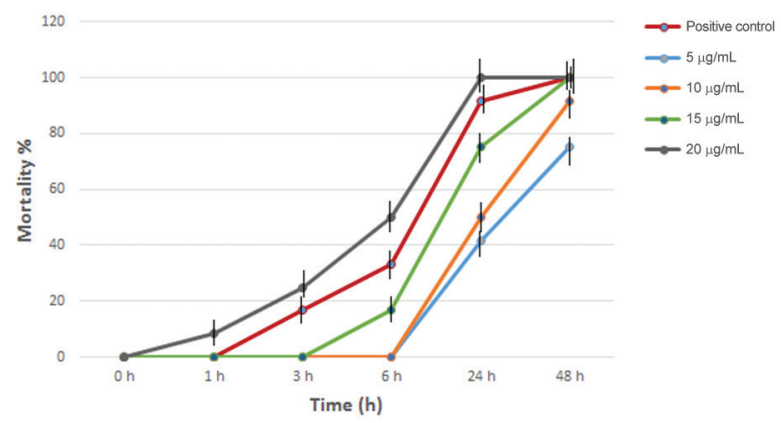

Fig. 2: mortality rate (\%) of Schistosoma mansoni adult male worms treated in vitro with different concentrations of primaquine for different incubation times. The mortality rate of both the negative control group and the group treated with $2 \mu \mathrm{g} / \mathrm{mL}$ of primaquine was $0 \%$. Error bars represent the \pm SD from three independent experiments.
PZQ at a concentration of $5 \mu \mathrm{g} / \mathrm{mL}$ showed slow motility with a mortality rate of $58.3 \%$ in juvenile worms, whereas contraction and paralysis with a mortality rate of $91.7 \%$ was observed in adult male worms after $24 \mathrm{~h}$ of incubation. These effects progressed and became more intense as the time of incubation increased until the motility of all worms was reduced. The worms were considered dead after $48 \mathrm{~h}$ of exposure (Figs 1-2).

The mortality rate of both juvenile and adult male worms of $S$. mansoni, exposed to primaquine was also studied. At the concentrations 2, 5, and $10 \mu \mathrm{g} / \mathrm{mL}$, the mortality rate in juvenile worms was $0 \%, 50 \%$, and $58.3 \%$, respectively after $24 \mathrm{~h}$ of incubation, whereas it was $0 \%, 83.3 \%$, and $100 \%$, respectively after $48 \mathrm{~h}$ of incubation. On the other hand, in adult male worms the mortality rate was $0 \%, 41.7 \%$, and $50 \%$, respectively after $24 \mathrm{~h}$ of incubation, whereas after $48 \mathrm{~h}$ of incubation it was $0 \%, 75 \%$, and $91.7 \%$, respectively. In addition, juvenile worms appeared slightly more sensitive to the schistosomicidal activity of primaquine than did the adult male worms at a concentration of $15 \mu \mathrm{g} / \mathrm{mL}$. Meanwhile, the concentration of $20 \mu \mathrm{g} / \mathrm{mL}$ caused contraction, paralysis, followed by death of all worms after $24 \mathrm{~h}$ of exposure to the drug (data not shown). Parasite death was irreversible, as judged by the examination of worms following washing and overnight incubation in drug free medium. Thus, the mortality rate of the worms was directly proportional to both the concentration and the period of incubation (Figs 1-2).

Tegumental alternations in S. mansoni juvenile and adult male worms in response to primaquine as visualised by SEM - Both juvenile (21 days old) and adult male $S$. mansoni worms (seven weeks old) incubated in pure medium (negative control) showed intact surface structure and topography (Fig. 3A-D). In the juvenile worm, the body consisted of oral and ventral suckers and a bore exhibited the beginning of the gynaecophoric groove (Fig. 3A), whereas the dorsal surface is covered
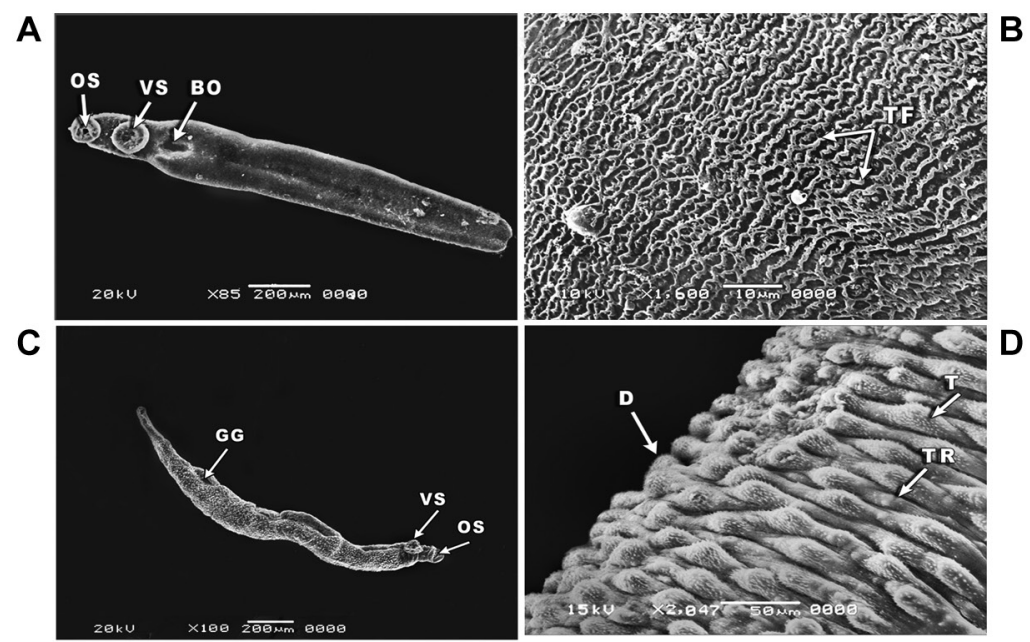

Fig. 3: scanning electron microscopy (SEM) of Schistosoma mansoni juvenile (A-B) and adult male worms (C-D) incubated in pure medium (negative control) for $24 \mathrm{~h}$ showing: (A) juvenile worm with an oral sucker (OS), ventral sucker (VS) and a bore (BO) marking the beginning of the gynaecophoric groove $(\times 85)$; (B) the dorsal surface of the juvenile worm showing rows of tegumental folds (TF) $(\times 1600)$; (C) adult male worm showing the oral sucker (OS), ventral sucker (VS), and gynaecophoric groove (GG) $(\times 100)$; (D) adult male worm with well-developed tubercles (T) and tegumental ridges (TR) on the dorsal surface (D) $(\times 2.047)$. 
A

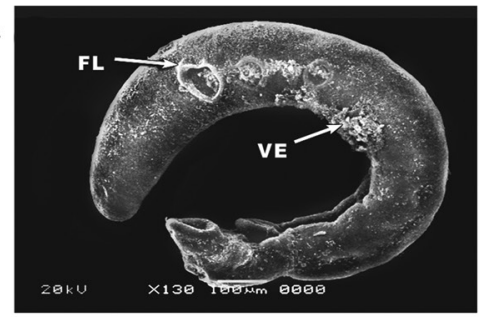

C

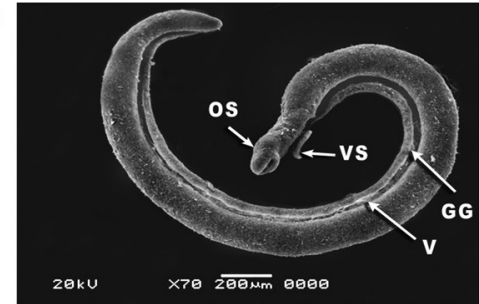

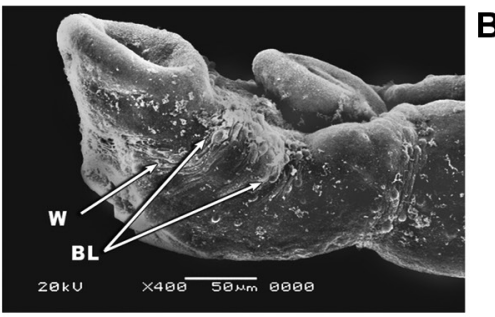

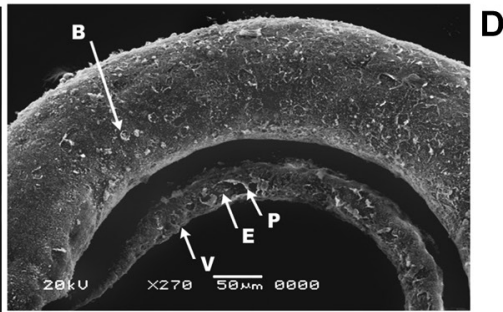

Fig. 4: scanning electron microscopy (SEM) of Schistosoma mansoni juvenile (A-B) and adult male worms (C-D) exposed to $5 \mu \mathrm{g} / \mathrm{mL}$ of Praziquantel (PZQ) (positive control) for $24 \mathrm{~h}$ showing: (A) juvenile worm contraction with vesicles (VE) and focal lesions (FL) in the middle part of body ( $\times 130)$; (B) magnification of the anterior region of a juvenile worm showing tegumental wrinkles (W) and blisters (BL) $(\times 400)$; (C) adult male worm bending in the ventral (V) direction with oral sucker (OS), ventral sucker (VS), and gynecophric groove (GG) $(\times 70)$; (D) ventral surface $(V)$ of an adult male worm showing peeling $(\mathrm{P})$ and erosion $(\mathrm{E})$ of the tegument with blebs $(\mathrm{B})(\times 270)$.

A

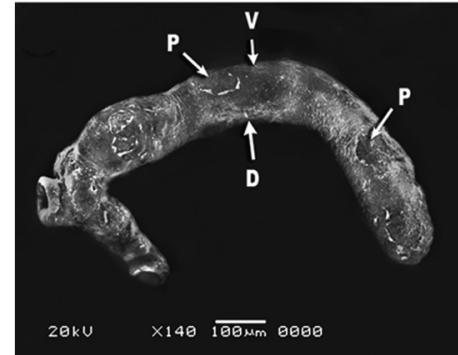

C

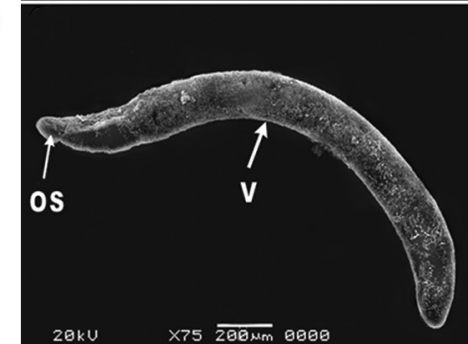

E

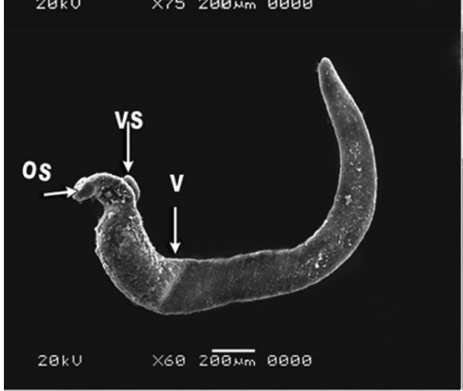

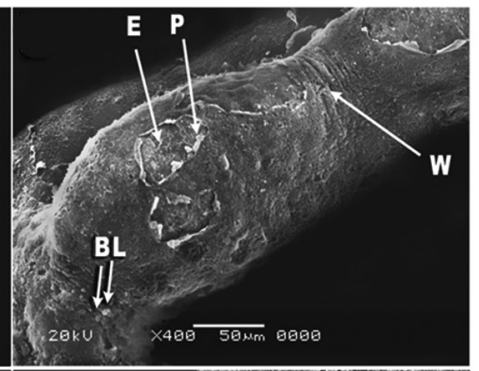
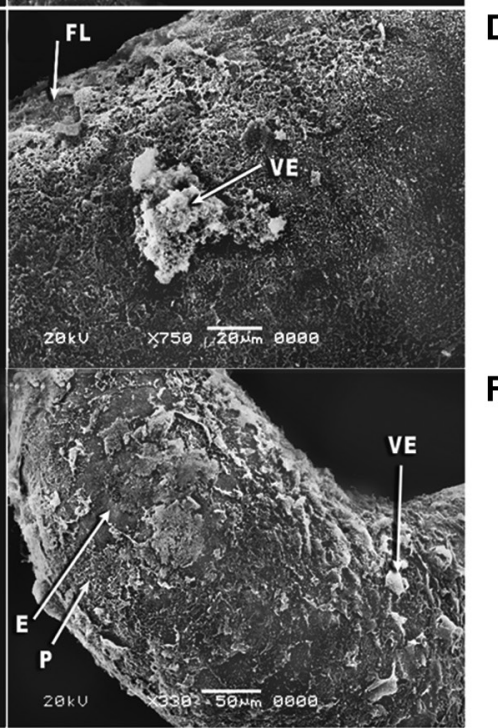

B

D

F

Fig. 5: scanning electron microscopy (SEM) of Schistosoma mansoni juvenile worms exposed to different concentrations of primaquine (A-F) for 24 h showing: (A) juvenile worm bending dorsally (D) with tegumental peeling $(\mathrm{P})$ on the ventral surface (V) of the worm at concentration $10 \mu \mathrm{g} / \mathrm{mL}(\times 140)$; (B) magnification of the anterior region of a juvenile worm showing erosion (E), peeling (P) and blisters (BL) with tegumental wrinkles $(\mathrm{W})$ at the concentration $10 \mu \mathrm{g} / \mathrm{mL}(\times 400)$; (C) juvenile worm with curving body in the ventral direction $(\mathrm{V})$ showing oral sucker $(\mathrm{OS})$ at the concentration $15 \mu \mathrm{g} / \mathrm{mL}(\times 75)$; (D) magnification of the anterior region of a juvenile worm showing focal lesions (FL) on the tegument with large vesicles (VE) at the concentration $15 \mu \mathrm{g} / \mathrm{mL}(\times 750)$; (E) juvenile worm with ventrally (V) bending body showing oral sucker (OS) and ventral sucker (VS) at the concentration $20 \mu \mathrm{g} / \mathrm{mL}(\times 60)$; (F) magnification of the anterior region of a juvenile worm showing erosion (E), peeling $(\mathrm{P})$, and vesicles (VE) at the concentration $20 \mu \mathrm{g} / \mathrm{mL}(\times 330)$. 
with rows of tegumental folds (Fig. 3B). The adult male worm (negative control) showed oral and ventral suckers and the gynaecophoric groove on the ventral surface (Fig. 3C), with well-developed tubercles and tegumental ridges on the dorsal surface (Fig. 3D).

Tegument alterations were observed on $S$. mansoni juvenile and adult male worms after $24 \mathrm{~h}$ of incubation with a concentration of $5 \mu \mathrm{g} / \mathrm{mL}$ PZQ (positive control) (Fig. 4A-D). In the juvenile worm, the morphological alternations were demonstrated as contraction of the worm body with vesicles and focal lesions in the middle region of the worm (Fig. 4A). In addition, some wrinkles and blisters appeared in the anterior area of the juvenile worm (Fig.
4B). In the adult male worm, the entire body was bent ventrally (Fig. 4C) and the tegument exhibited extensive areas of peeling and erosion with blebs (Fig. 4D).

Twenty-four hours after exposure to primaquine at different concentrations, all the examined juvenile and adult male worms showed variable degrees of tegumental changes. Most of the juvenile worms incubated at the concentration of $10 \mu \mathrm{g} / \mathrm{mL}$ were characterised by dorsal bending of the worm body with tegumental peeling on the ventral surface (Fig. 5A). Moreover, the magnified anterior portion of the worm showed some erosion, peeling, blisters, and wrinkles (Fig. 5B). Additionally, at the concentration $15 \mu \mathrm{g} / \mathrm{mL}$, the worms

A
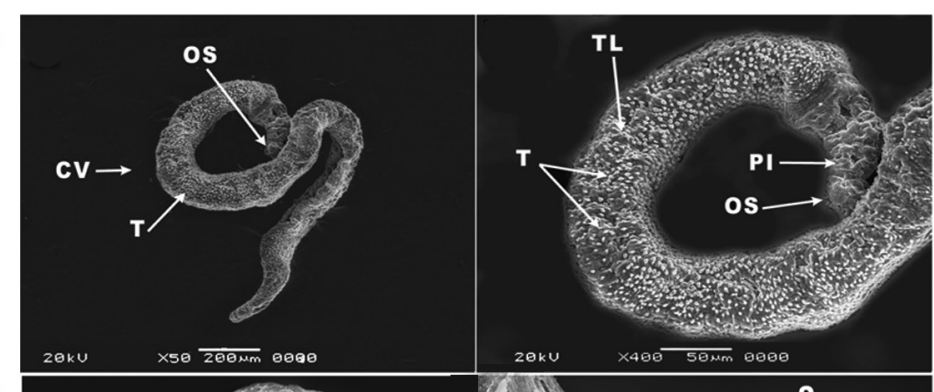

C
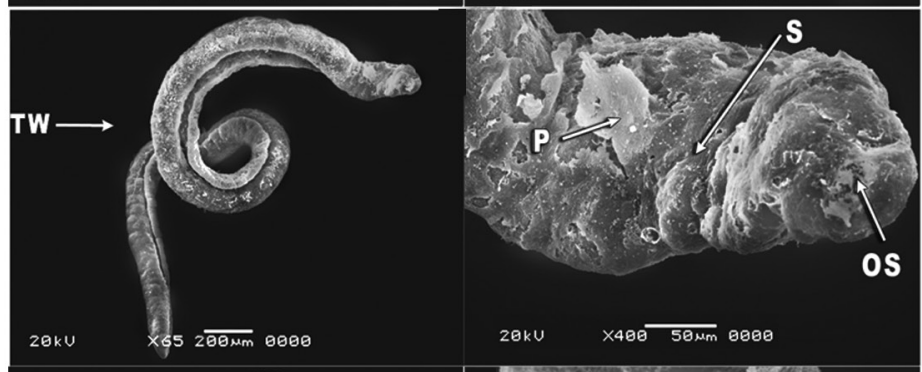

E
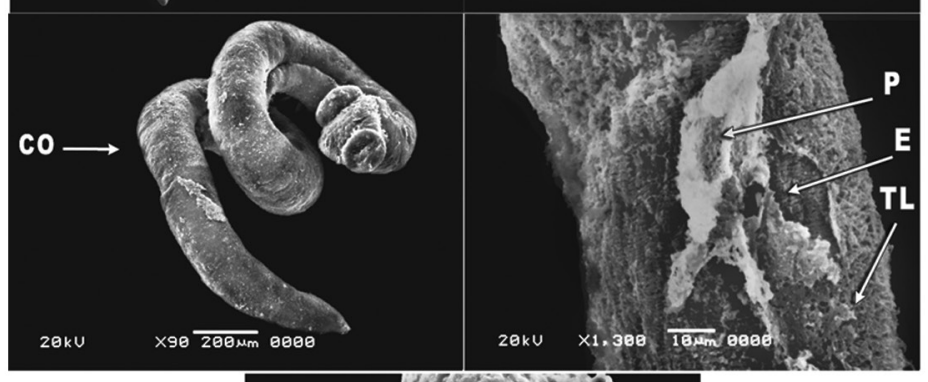

\section{B}

D

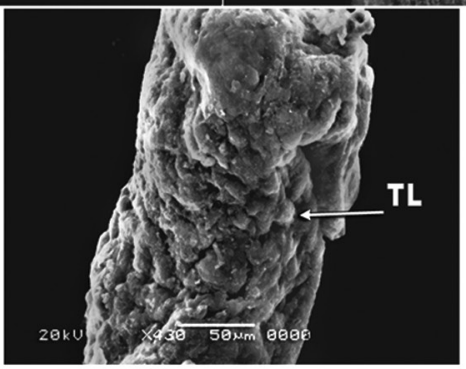

G

Fig. 6: scanning electron microscopy (SEM) of Schistosoma mansoni adult male worms exposed to different concentrations of primaquine (A-G) for $24 \mathrm{~h}$ showing: (A) convoluting (CV) of the adult male worm body with destruction of tubercles (T) at the concentration $10 \mu \mathrm{g} / \mathrm{mL}(\times 50)$; (B) magnification of the anterior region of an adult male worm showing pitted (PI) tegumental layer (TL), destruction of tubercles (T) and changes in the oral sucker (OS) at the concentration $10 \mu \mathrm{g} / \mathrm{mL}(\times 400)$; (C) twisting (TW) of the adult male worm at the middle region of the body at the concentration $15 \mu \mathrm{g} / \mathrm{mL}$ ( $\times 65$ ); (D) magnification of the anterior region of adult male worm showing destruction of the oral sucker (OS) and sloughing (S) of the tegument with peeling $(\mathrm{P})$ at the concentration $15 \mu \mathrm{g} / \mathrm{mL}(\times 400)$; (E) adult male worm showing coiling body (CO) at the concentration $20 \mu \mathrm{g} /$ $\mathrm{mL}(\times 90)$; (F) magnification of the middle region of an adult male worm showing extensive peeling (P) and erosion (E) of the tegumental layer (TL) at the concentration $20 \mu \mathrm{g} / \mathrm{mL}(\times 1300)$; (G) adult male worm with destruction of the tegumental layer (TL) at the concentration $20 \mu \mathrm{g} / \mathrm{mL}(\times 430)$. 
were curved ventrally and their anterior region showed focal lesions on the tegument with large vesicles (Fig. $5 \mathrm{C}$-D). Furthermore, severe damages were observed at the concentration $20 \mu \mathrm{g} / \mathrm{mL}$, where the worm was bent ventrally (Fig. 5E) with extensive erosion and peeling that extended along the entire dorsal worm surface as along with vesicles (Fig. 5F). In case of adult male worms incubated with primaquine at the concentration $10 \mu \mathrm{g} / \mathrm{mL}$ for $24 \mathrm{~h}$, the worm body was convoluted with marked destruction of tubercles (Fig. 6A). Besides the pitting of the tegumental layer, changes in oral sucker were also observed (Fig. 6B). At the concentration 15 $\mu \mathrm{g} / \mathrm{mL}$, the worms showed twisting in the middle region along with destruction of the oral sucker and sloughing of the tegument (Fig. 6C-D). Additionally, coiling of the male worm body was observed at the concentration 20 $\mu \mathrm{g} / \mathrm{mL}$ (Fig. 6E), with extensive peeling and erosion of the tegumental layer (Fig. 6F). Severe destruction of the tegumental layer was also observed (Fig. 6G). However, at low concentrations ( 2 and $5 \mu \mathrm{g} / \mathrm{mL}$ ) no morphological alterations were observed. Thus, the specific morphological changes in juvenile and adult male worms were closely related to the drug concentration used.
Effect of primaquine on the ultrastructural alternations of the S. mansoni adult male worm tegument using transmission electron microscopy (TEM) - Since the ultrastructure of the tegument of juvenile $S$. mansoni worms was more or less similar to that of adult worms, the ultrastructural alterations in the tegument of S. mansoni adult male worms were studied. In pure medium (negative control), the adult male S. mansoni worm tegument contained numerous normal pointed spines with their triangular shaped covering the tegumental layer; normal muscles were also monitored in the subtegumental layer (Fig. 7A). The tegument of the S. mansoni adult male worm incubated with PZQ (positive control) for 24 $\mathrm{h}$, revealed noticeable degeneration of the tegumental and subtegumental layers, embedded spines, and muscle shrinkage with widespread vacuolisation (Fig. 7B).

The tegumental surface of adult male worms incubated with different concentrations of primaquine showed damaged tegumental structure that varied from slight to severe. At $10 \mu \mathrm{g} / \mathrm{mL}$ of primaquine, some spines were embedded and some others were still pointed with disorganisation of the underlying muscles, accompanied by many small vacuoles (Fig. 7C). Moreover, more spines

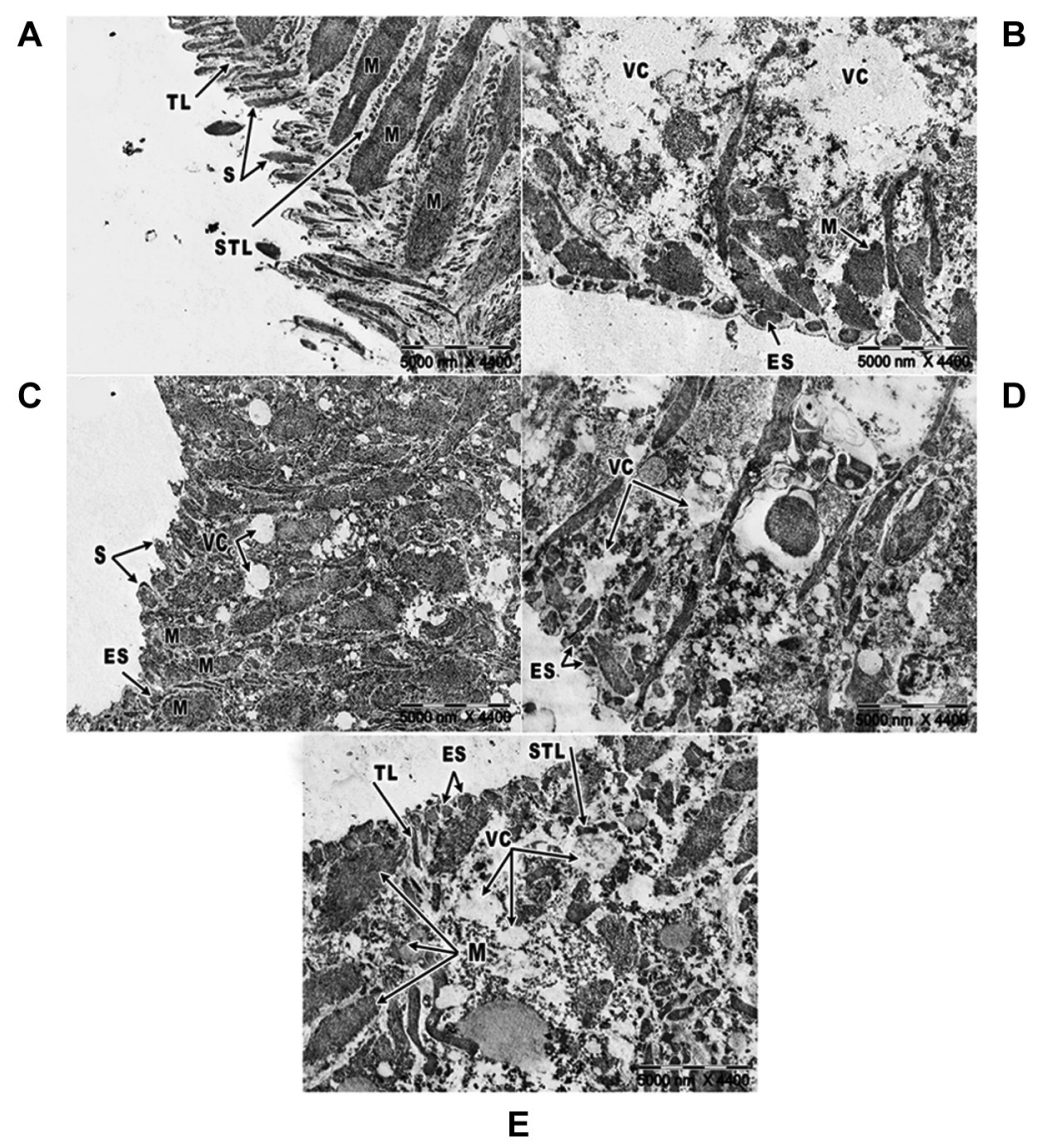

Fig. 7: ultrastructural alterations in adult male Schistosoma mansoni worms: (A) Adult male worm incubated for 24 h in pure medium (negative control) showing normal pointed spines (S), tegumental layer (TL), subtegumental layer (STL), and normal muscles (M) $(\times 4400)$; (B) adult male worm maintained in a medium with Praziquantel (PZQ) (positive control) for 24 h showing embedded spines (ES) and shrinkage in muscles (M) with formation of vacuoles (VC) $(\times 4400)$; (C) adult male worm exposed to primaquine for 24 h showing embedded spines (ES) and pointed spines (S) with small vacuoles (VC) between the normal muscles (M) at the concentration $10 \mu \mathrm{g} / \mathrm{mL}(\times 4400)$; (D) more embedded spines (ES) and a large vacuole (VC) in adult male worm at the concentration $15 \mu \mathrm{g} / \mathrm{mL}(\times 4400)$; (E) adult male worm with degeneration in both the tegumental (TL) and subtegumental layers (STL), embedded spines (ES), large vacuoles (VC), and shrinkage in the muscles $(\mathrm{M})$ at the concentration $20 \mu \mathrm{g} / \mathrm{mL}(\times 4400)$. 
were completely embedded in the tegumental layer with more degeneration in the subtegumental layer and large vacuoles at the concentration $15 \mu \mathrm{g} / \mathrm{mL}$ (Fig. 7D). Increasing the concentration resulted in more alterations. At a high concentration $(20 \mu \mathrm{g} / \mathrm{mL})$, extensive damage of the tegumental and subtegumental layers with embedded spines was observed (Fig. 7E).

\section{DISCUSSION}

Schistosomiasis is treated by the administration of PZQ, which exhibits good efficacy and low toxicity. Despite this, $S$. mansoni shows drug tolerance or resistance (Doenhoff et al. 2008). Therefore, it is important to develop new drugs for treating this disease, since PZQ is far from ideal (Lima et al. 2011). Recent discoveries have shown that the anti-malarial drug primaquine exhibited schistosomicidal activity against both juvenile and adult worms of $S$. mansoni in vitro (Holtfreter et al. 2011). The anti-schistosomal effects of primaquine are noteworthy as it is used for the treatment of malaria in schistosomiasis endemic areas (Holtfreter et al. 2011).

In this study, adult male worms were used instead of females, because females are not in direct contact with the host microenvironment. Earlier studies have shown that soft tissue alterations in male worms are more pronounced than those in female worms (Mostafa \& Soliman 2002, Matos-Rocha et al. 2016).

Both scanning and transmission electron microscope play an important role in elucidating the detailed morphology and different alterations of the S. mansoni tegument allowing the interpretation of its functionality (Senft \& Gibler 1977, El-Shabasy et al. 2015). These methods have been employed by several researchers (de Oliveira et al. 2012, El-Shabasy et al. 2015) and help to explain the mechanisms of action of anti-schistosomal drugs. The normal S. mansoni tegument is an essential interface between the parasite and the intravascular environment in the host (El-Shabasy et al. 2015).

Juvenile worms incubated with PZQ at $5 \mu \mathrm{g} / \mathrm{mL}$ for $24 \mathrm{~h}$ showed slow motion with a mortality rate of $58.3 \%$. Moreover, the body was contracted with vesicles and focal lesions, as well as wrinkles and blisters. However, in the adult $S$. mansoni male worms, absence of movement as well as paralysis of the parasites was observed with a mortality rate of $91.7 \%$. Moreover, the whole worm body was bent ventrally and the tegument exhibited extensive areas of peeling and erosion with blebs. These results were in accordance with those reported by Pica-Mattoccia and Cioli (2004), who demonstrated that PZQ caused both contractions and paralysis in adult worms of $S$. mansoni with little effect on juvenile worms at the concentrations 0.1 and $1 \mu \mathrm{g} / \mathrm{mL}$. PZQ damaged the tegument, and facilitated eosinophilic granulocytes to damage the parasites. In addition, it led to contraction and paralysis accompanied by muscle spasms in the worm, caused by the influx of $\mathrm{Ca}^{2+}$ ions into the tegument (Shaw \& Erasmus 1987). These finding are in parallel with those reported by Mehlhorn et al. (1981), who stated that the principle effect of PZQ finally led to the death of $S$. mansoni with disruption of the worm tegument. Additionally, the ultrastructural changes in adult male worms included degeneration of the tegument and subtegumental layers as well as shrinkage of the muscle with embedded spines. These findings were in accordance with those of El-Shabasy et al. (2015) who confirmed the same ultrastructural transformation of the tegument induced by PZQ.

This study revealed that the effects of primaquine were dose-dependent, and the most effective dose was $20 \mu \mathrm{g} / \mathrm{mL}$ in a short incubation period of $24 \mathrm{~h}$. In addition, it was possible to observe that both juvenile and adult male worms exposed to the drug exhibited contraction, motility reduction, and paralysis resulting in death. The main changes induced by primaquine in the present study were at the concentrations 10,15 , and $20 \mu \mathrm{g} /$ $\mathrm{mL}$. In the juveniles, most of the changes included bending of the worm with tegumental erosion and peeling. Besides, blebs and wrinkles that extended to the entire dorsal surface of the worms as well as vesicles were also observed. The tegumental alternations in the adult male worms included convoluting of the worms, destruction of tubercles, and peeling and erosion of the tegumental layer. Furthermore, at low concentrations ( 2 and $5 \mu \mathrm{g} /$ $\mathrm{mL}$ ) no morphological alterations occurred. Mitsui and Aoki (2010) noticed that when $S$. mansoni adult worms were exposed to primaquine in vitro at the concentration $10 \mu \mathrm{g} / \mathrm{mL}$, the survival time of the adult worm was reduced with the appearance of remarkable blebs on the body of the male worm accompanied by swelling and finally death. Similar results were observed by Holtfreter et al. (2011), who elucidated the damage caused by primaquine in vitro on adult worms of $S$. mansoni. This damage was manifested in loss of their attachment to the walls of the culture plates, possibly due to the damage of the muscles in both the oral and ventral suckers.

The present study showed that $24 \mathrm{~h}$ after incubation in primaquine at the concentrations 10,15 , and $20 \mu \mathrm{g} /$ $\mathrm{mL}$, severe ultrastructural damage occurred in adult male worms. The highest degree of damage was seen in both the tegumental and subtegumental layers. The tegument layer was embedded with spines, whereas the subtegumental layer showed shrinkage of muscles with vacuoles at the concentrations 10 and $15 \mu \mathrm{g} / \mathrm{mL}$; thereafter, increasing the concentration to $20 \mu \mathrm{g} / \mathrm{mL}$ resulted in extensive damage including degeneration of both the tegumental and subtegumental layers.

There are no recent published studies concerning the ultrastructural changes induced by primaquine on $S$. mansoni adult male worms; the results of both scanning and transmission electron microscopy in the present work show that the schistosome tegument is the main target of primaquine. Several researchers have reported that the worm tegument is the key target for many antischistosomal drugs as mefloquine and Synriam ${ }^{\mathrm{TM}}$ (Manneck et al. 2010, Mossallam et al. 2015). In vitro assays cannot cover all aspects of drug anthelminthic activities, particularly pharmacological and immunological host interactions; however, they provide first evidence of anthelminthic effects and an insight into the mode of action, and they may lead to the development of new therapeutic drugs (Holtfreter et al. 2010). Considering the results obtained in this study, the schistosomicidal activity of pri- 
maquine indicates that this drug possesses moderate in vitro activity against juvenile and adult male $S$. mansoni worms, since it caused high schistosome mortality and pronounced morphological alterations. Therefore, this study opens up perspectives for future research on this drug in vivo to discover its mechanism of action.

\section{AUTHORS' CONTRIBUTIONS}

ROAK and FEAB were responsible for the study conception and design and the drafting of the manuscript, performed the data collection and data analysis, made critical revisions and final approval of the version to the paper to be published.

\section{REFERENCES}

Carneiro-Santos P, Thornhill JA, Doenhoff MJ, Hagan P, Kusel JR. Acidic vesicles of Schistosoma mansoni. Parasitol Res. 2001; 87(12): 1001-6.

Cheng L, Lei L, Guo S, Zhu C, Rong H, Guo D, et al. Schistosoma japonicum. Treatment of different developmental stages in mice with longacting praziquantel implants. Exp Parasitol. 2011; 129(3): 254-9.

Clegg JA, Smithers SR. The effects of immune rhesus monkey serum on schistosomula of Schistosoma mansoni during cultivation in vitro. Int J Parasitol. 1972; 2(1): 79-98.

Colley DG, Bustinduy AL, Secor WE, King CH. Human schistosomiasis. Lancet. 2014; 383(9936): 2253-64.

de Oliveira RN, Rehder VLG, Oliveira ASS, Montanari JI, Jeraldo VL, Linhares AX, et al. Schistosoma mansoni: in vitro schistosomicidal activity of essential oil of Baccharis trimera (Less) DC. Exp Parasitol. 2012; 132(2): 135-43.

Doenhoff MJ, Cioli D, Utzinger J. Praziquantel: mechanisms of action, resistance and new derivatives for schistosomiasis. Curr Opin Infect Dis. 2008; 21(6): 659-67.

Doenhoff MJ, Pica-Mattoccia L. Praziquantel for the treatment of schistosomiasis: its use for control in areas with endemic disease and prospects for drug resistance. Expert Rev Anti Infect Ther. 2006; 4(2): 199-210.

Duvall RH, DsWitt WB. An improved perfusion technique for recovering adult schistosomes from laboratory animals. Am J Trop Med Hyg. 1967; 16(4): 483-6.

El Bardicy S, El Sayed I, Yousif F, Van der Veken P, Haemers A, Augustyns K, et al. Schistosomicidal and molluscicidal activities of amino alkylamino substituted neo- and norneocryptolepine derivatives. Pharm Biol. 2012; 50(2): 134-40.

Elmes NJ1, Bennett SM, Abdalla H, Carthew TL, Edstein MD. Lack of sex effect on the pharmacokinetics of primaquine. Am J Trop Med Hyg. 2006; 74(6): 951-2.

El-Shabasy EA, Reda ES, Abdeen SH, Said AE, Ouhtit A. Transmission electron microscopic observations on ultrastructural alterations in Schistosoma mansoni adult worms recovered from C57BL/6 mice treated with radiation attenuated vaccine and/or praziquantel in addition to passive immunization with normal and vaccinated rabbit sera against infection. Parasitol Res. 2015; 114(4): 1563-80.

Holtfreter MC, Loebermann M, Frei E, Riebold D, Wolff D, Hartung DD, et al. Schistosomula, pre-adults and adults of Schistosoma mansoni ingest fluorescence-labelled albumin in vitro and in vivo: implication for a drug-targeting model. Parasitology. 2010; 137(11): 1645-52.

Holtfreter MC, Loebermann MCM, Klammt S, Sombetzki M, Bodammer P, Riebold D, et al. Schistosoma mansoni: schistosomicidal effect of mefloquine and primaquine in vitro. Exp Parasitol. 2011; 127(1): 270-6.

Lima CMBL, Freitas FID, de Morais LCSL, Cavalcanti MGS, da Silva LF, Padilha RJR, et al. Ultrastructural study on the morphologi- cal changes to male worms of Schistosoma mansoni after in vitro exposure to allicin. Rev Soc Bras Med Trop. 2011; 44(3): 327-30.

Manneck T, Haggenmüller Y, Keiser J. Morphological effects and tegumental alterations induced by mefloquine on schistosomula and adult flukes of Schistosoma mansoni. Parasitology. 2010; 137(1): 85-98.

Mata-Santos HA, Dutra FF, Rocha CC, Lino FG, Xavier FR, Chinalia LA, et al. Silymarin reduces profibrogenic cytokines and reverses hepatic fibrosis in chronic murine schistosomiasis. Antimicrob Agents Chemother. 2014; 58(4): 2076-83.

Matos-Rocha TJ, Cavalcanti MGS, Veras DL, Feitosa APS, Goncalves GGA, Portela-Junior NC, et al. Ultrastructure Changes in Schistosoma mansoni male worms after in vitro incubation with essential oil Menthax villosa Huds. Rev Inst Med Trop São Paulo. 2016; 58(4): 4-6.

Mehlhorn H, Becker B, Andrews P, Thomas H, Prenkel JK. In vitro and in vivo experiments on the effects of praziquantel on Schistosoma mansoni. A light and electron microscopy study. Arzneimittelforschung. 1981; 31(3a): 544-4.

Mitsui Y, Aoki Y. In vitro effects of current antimalarial drugs on the survival of paired Schistosoma mansoni adult worms and their egg production. Trop Med Health. 2010; 38: 69-73.

Moraes J. Antischistosomal natural compounds: present challenges for new drug screens. In: Rodriguez-Morales AJ, editor. Current topics in tropical medicine. Rijeka: Intech; 2012. p. 333-58.

Mossallam SF, Amer EI, El-Faham MH. Efficacy of Synriam ${ }^{\text {TM }}$, a new antimalarial combination of OZ277 and piperaquine against different developmental stages of Schistosoma mansoni. Acta Trop. 2015; 143: 36-46.

Mostafa OM, Soliman MI. Experimental use of black-seed oil against Schistosoma mansoni in albino mice: II. Surface topography of adult worms. Egypt J Med Lab Sci. 2002; 11(1): 79-85.

Pereira AC, Silva MLA, Souza JM, de Laurentiz RS, Rodrigues V, Januário $\mathrm{AH}$, et al. In vitro and in vivo anthelmintic activity of (-)-6,6'-dinitrohinokinin against schistosomula and juvenile and adult worms of Schistosoma mansoni. Acta Trop. 2015; 149: 195-201.

Pica-Mattoccia L, Cioli D. Sex and stage-related sensitivity of Schistosoma mansoni to in vivo and in vitro praziquantel treatment. Int J Parasitol. 2004; 34(4): 527-33.

Senft AW, Gibler WB. Schistosoma mansoni tegumental appendages: scanning microscopy following thiocarbohydrazide-osmium preparation. Am J Trop Med Hyg. 1977; 26: 1169-77.

Shaw MK, Erasmus DA. Schistosoma mansoni: structural damage and tegumental repair after in vivo treatment with praziquantel. Parasitology. 1987; 94(2): 243-54.

Xavier AML, Magalhães JAS, Cunha GDP, Silva AC, Tavares DAG, Sarro-Silva MD, et al. Morphological tegument alterations of adult Schistosoma mansoni harbored in non antihelminthic treated, high-immune-tolerogenic and low-inflammatory mice. Acta Trop. 2010; 116(1): 95-9.

Xavier AML, Tavares D, Guimarães EV, Sarro-Silva MD, Silva AC, Neto A. Ultrastructural alterations in adult Schistosoma manso$n i$, harbored in non-antihelminthic treated and low-inflammatory mice by transmission electron microscopy (TEM). Acta Trop. 2014; 130(1): 51-7.

Xiao S, Qiao C, Xue J, Wang L. Mefloquine in combination with hemin causes severe damage to adult Schistosoma japonicum in vitro. Acta Trop. 2014; 131: 71-8.

Xiao SH, Keiser J, Chollet J, Utzinger J, Dong Y, Endriss Y, et al. In vitro and in vivo activities of synthetic trioxolanes against major human schistosome species. Antimicrob Agents Chemother. 2007; 51(4): 1440-5. 